\title{
The effect of exergaming on knee proprioception in older men: A randomized controlled trial
}

\begin{abstract}
Background: Proprioception is the ability to sense the body position, muscle sense, joint stability and posture. As balance decreases during the process of aging, knee proprioception has a critical role in body balance and daily activities. Exergaming has shown to be a potentially effective and more enjoyable form of exercise delivery.

Objective: The purpose of this study was to determine the effect of an 8-week Xbox Kinect exercise program on knee proprioception in healthy older adults.

Methodology: Thirty-two elderly men who were 65 years of age or older were randomly allocated to either a control or experimental group (allocation ratio 1:1). The experimental group received an exergame intervention that included Xbox Kinect with games focusing on movements of the knee joint for 8 weeks (three times per week and 40 min per sessions). A Biodex Isokinetic Dynamometer was used to measure knee joint position sense before and after the exercise program.

Results: After eight weeks of training, knee proprioception significantly improved in the intervention group for several knee joint angles: $30^{\circ}(3.5 \pm 1.1), 45^{\circ}(3.1 \pm 0.9)$, and $60^{\circ}(3.0$ $\pm 0.6)$ compared to the control group $30^{\circ}(5.2 \pm 0.8), 45^{\circ}(5.2 \pm 0.8)$, and $60^{\circ}(6.2 \pm 0.9)$ (dominant leg F1, $28=23.469, \mathrm{p}=0.001$. $\mathrm{\eta} 2=0.456$; non-dominant leg F1, $28=23.076, \mathrm{p}=$ 0.001. $\eta 2=0.452)$.
\end{abstract}

Conclusion: The results from this study indicate that exergame intervention can enhance knee proprioception in elderly men.

Keyword: Aging; Falling; Knee proprioception; Exergame 